\title{
Synthesis, characterization and bio-activity of S-2-picolyldithiocarbazate (S2PDTC), some of its Schiff bases and their Ni(II) complexes and X-ray structure of S-2-picolyl- $\beta$ - $\mathrm{N}$-(2-acetylpyrrole)dithiocarbazate
}

\begin{abstract}
A new dithiocarbazate ligand, S-2-picolyldithiocarbazate (S2PDTC) was synthesized using 2picolylchloride hydrochloride. Tridentate Schiff bases were prepared by condensation of S-2picolyldithiocarbazate (S2PDTC) with pyridine-2-carboxaldehyde (NNSNlj 2-acetylpyrrole (NNSnj and 2-acetylthiophene (NSS), while a bidentate Schiff base (NS) was prepared by condensing the S2PDTC with 2-acetylfuran. Complexes of S2PDTC and its Schiff bases with $\mathrm{Ni}$ (II) salts were synthesized and characterized by elemental analyses and various physicochemical techniques. A square-planar structure has been proposed for the diamagnetic [Ni(S2PDTC)2] and [Ni(NNSnj · Cl] complexes while [Ni(NS) - Cl] complex was dimeric. Complexes of [Ni(NNS) $($ f $]$ and [Ni(NSS)2] were paramagnetic with octahedral stereochemistry. S2PDTC showed activity against bacteria and fungi (inhibitory zones above $15 \mathrm{~mm}$ ). The NS and NSS Schiff bases showed activity toward a number of the bacteria assayed, while the NS and NSS Schiff bases, and the [Ni(NNSnj $\cdot \mathrm{Cl}]$ and $[\mathrm{Ni}(\mathrm{NS}) \cdot \mathrm{Cl}]$ complexes were found to be active only against C. lypolytica (2075). S2PDTC proved moderately active against HT-29 and weakly active toward CEM-SS with CD50 values of 9.5 and $24.0 \mathrm{\varepsilon g} \mathrm{cmī} \mathrm{3,} \mathrm{respectively,} \mathrm{while} \mathrm{among} \mathrm{its} \mathrm{Schiff} \mathrm{bases} \mathrm{reported} \mathrm{herein,} \mathrm{only} \mathrm{the} \mathrm{NNSNj}$ Schiff base showed strong activity toward CEM-SS (Human cell T-lymphoblastic leukemia) and HT-29 (Human colon adenocarcinoma cells) with CD50 values of $2.3 \mathrm{\varepsilon g} \mathrm{cmī} \mathrm{3.} \mathrm{All} \mathrm{of}$ the $\mathrm{Ni}(\mathrm{II})$ complexes were inactive against CEM-SS cancer cells.
\end{abstract}

Keyword: S-2-picolyldithiocarbazate (Hydrazine carbodithioic acid pyridinium-2-yl methyl ester chloride); Bidentate NS Schiff base; Tridentate NNS and NSS Schiff bases; Ni(II) complexes; Dithiocarbazate derivative 